PROCEEDINGS OF THE

AMERICAN MATHEMATICAL SOCIETY

Volume 127, Number 12, Pages 3721-3728

S 0002-9939(99)04981-3

Article electronically published on May 17, 1999

\title{
ON A PROBLEM OF DYNKIN
}

\author{
YUAN-CHUNG SHEU
}

(Communicated by Stanley Sawyer)

\begin{abstract}
Consider an $(L, \alpha)$-superdiffusion $X$ on $\mathbb{R}^{d}$, where $L$ is an uniformly elliptic differential operator in $\mathbb{R}^{d}$, and $1<\alpha \leq 2$. The $\mathbb{G}$-polar sets for $X$ are subsets of $\mathbb{R} \times \mathbb{R}^{d}$ which have no intersection with the graph $\mathbb{G}$ of $X$, and they are related to the removable singularities for a corresponding nonlinear parabolic partial differential equation. Dynkin characterized the $\mathbb{G}$-polarity of a general analytic set $A \subset \mathbb{R} \times \mathbb{R}^{d}$ in term of the Bessel capacity of $A$, and Sheu in term of the restricted Hausdorff dimension. In this paper we study in particular the $\mathbb{G}$-polarity of sets of the form $E \times F$, where $E$ and $F$ are two Borel subsets of $\mathbb{R}$ and $\mathbb{R}^{d}$ respectively. We establish a relationship between the restricted Hausdorff dimension of $E \times F$ and the usual Hausdorff dimensions of $E$ and $F$. As an application, we obtain a criterion for $\mathbb{G}$-polarity of $E \times F$ in terms of the Hausdorff dimensions of $E$ and $F$, which also gives an answer to a problem proposed by Dynkin in the 1991 Wald Memorial Lectures.
\end{abstract}

\section{INTRODUCTION}

Suppose that $L$ is an uniformly elliptic differential operator in $\mathbb{R} \times \mathbb{R}^{d}$ of the form

$$
L u(t, x)=\sum_{i, j} a_{i j}(t, x) \frac{\partial^{2} u}{\partial x_{i} \partial x_{j}}+\sum_{i} b_{i}(t, x) \frac{\partial u}{\partial x_{i}} .
$$

Here we assume that $a_{i j}$ and $b_{i}$ are bounded and smooth functions in $\mathbb{R} \times \mathbb{R}^{d}$. An $(L, \alpha)$-superdiffusion, $1<\alpha \leq 2$, is a branching measure-valued Markov process $X=\left(X_{t}, P_{\mu}\right)$ such that for every bounded positive Borel function $f$ on $\mathbb{R}^{d}$, the function

$$
v(r, x)=-\log P_{\delta_{r, x}} e^{-\left\langle f, X_{t}\right\rangle}
$$

is a mild solution of the following problem:

$$
\begin{cases}\frac{\partial v}{\partial t}+L v & =v^{\alpha} \text { in }(-\infty, t) \times \mathbb{R}^{d}, \\ v(r, x) & \rightarrow f(x) \text { as } r \uparrow t \text { and } x \in \mathbb{R}^{d} .\end{cases}
$$

(Here we write $P Y$ for the expected value of $Y$ with respect to the probability measure $P$, and $\langle f, \mu\rangle$ for the integral of $f$ with respect to the measure $\mu$.) The graph of $X$ is the minimal closed subset $\mathbb{G}$ of $\mathbb{R} \times \mathbb{R}^{d}$ such that, for every $t \in \mathbb{R}$,

Received by the editors December 1, 1997 and, in revised form, February 23, 1998.

1991 Mathematics Subject Classification. Primary 60J60, 35K55; Secondary 60J80, 31C45.

Key words and phrases. Superdiffusion, graph of superdiffusion, semilinear partial differential equation, $\mathbb{G}$-polarity, $\mathbb{H}$-polarity, Hausdorff dimension, box dimension, restricted Hausdorff dimension. 
the measure $X_{t}$ is concentrated on the $t$-section of $\mathbb{G}$ An analytic set $A$ of $\mathbb{R} \times \mathbb{R}^{d}$ is called $\mathbb{G}$-polar if for every $r<t$ and $x \in \mathbb{R}^{d}$, we have

$$
P_{\delta_{r, x}}\left(\mathbb{G} \cap A_{t}=\emptyset\right)=1,
$$

where $A_{t}=A \cap\left([t, \infty) \times \mathbb{R}^{d}\right)$. In [2], Dynkin proved that the class of $\mathbb{G}$-polar sets for the $(L, \alpha)$-superdiffusion $X$ is identical to the class of sets of Bessel capacity zero. Moreover he proved that a set $A$ is $\mathbb{G}$-polar if and only if it is a removable singularity for the partial differential equation

$$
\frac{\partial v}{\partial t}+L v=v^{\alpha} .
$$

(We say that $A \subset \mathbb{R} \times \mathbb{R}^{d}$ is a removable singularity for the equation (1) if 0 is the only nonnegative solution of the equation (1) in $\left(\mathbb{R} \times \mathbb{R}^{d}\right) \backslash A$.) In [7], Sheu demonstrated that the critical restricted Hausdorff dimension (to be introduced later) for the $\mathbb{G}$-polarity is $d-\frac{2}{\alpha-1}$.

We say that an analytic set $F$ in $\mathbb{R}^{d}$ is $\mathbb{H}$-polar if $\{t\} \times F$ is $\mathbb{G}$-polar for every $t \in \mathbb{R}$. The notion of $\mathbb{H}$-polarity is also related to solutions of the heat equation with initial measure value. (See Baras and Pierre [1].) Note that the critical Hausdorff dimension for the $\mathbb{H}$-polarity is $d-\frac{2}{\alpha-1}$.

Our objective is to study the following problem proposed by Dynkin in the 1991 Wald Memorial Lectures.

Problem ([3], p. 1245). Suppose $F$ is $\mathbb{H}$-polar and $E \subset \mathbb{R}$ is a set of Lebesgue measure 0 . Is $E \times F \mathbb{G}$-polar?

In Section 2 we recall definitions of Hausdorff dimension, box dimension and the restricted Hausdorff dimension, and establish new relations between the restricted Hausdorff dimension of $E \times F$ and the Hausdorff dimensions of $E$ and $F$. Namely, we prove that

$$
\begin{aligned}
2 H-\operatorname{dim}(E)+H-\operatorname{dim}(F) & \leq \mathcal{R}-H-\operatorname{dim}(E \times F) \\
& \leq 2 H-\operatorname{dim}(E)+B-\operatorname{dim}_{-}(F) .
\end{aligned}
$$

where $H$-dim means the Hausdorff dimension, $B$ - dim - the lower Hausdorff box dimension, and $\mathcal{R}-H$-dim the restricted Hausdorff dimension. (Our proofs are analogous (with some suitable modifications) to that of Falconer [4] and from there we also quote some interesting examples.) Under the assumption $H$ - $\operatorname{dim}(F)=$ $B$ - $\operatorname{dim}_{-}(F)$, we obtain that if $2 H-\operatorname{dim}(E)+H$ - $\operatorname{dim}(F)<d-\frac{2}{\alpha-1}$, then $E \times F$ is $\mathbb{G}$-polar, whereas, if $2 H-\operatorname{dim}(E)+H-\operatorname{dim}(F)>d-\frac{2}{\alpha-1}$, then $E \times F$ is not $\mathbb{G}$-polar. As an application, we give examples in Section 3 which show that the answer to Dynkin's problem is negative (see Theorem 6 for more details).

\section{HAUSDORFF DIMENSION, BOX DIMENSION AND THE RESTRICTED HAUSDORFF DIMENSION}

Suppose that $F$ is a subset of $\mathbb{R}^{d}$. First we recall a definition of the Haudorff dimension of $F$. For every $s>0$ and $\epsilon>0$, set

$$
\wedge^{s}-m_{\epsilon}(F)=\inf \sum_{i}\left(\operatorname{diam}\left(B_{i}\right)\right)^{s}
$$


where the infimum is taken over all countable coverings of $F$ by open ball $B_{i}$ with radius $r_{i}<\epsilon$. The Hausdorff measure with index $s$ is defined by the formula

$$
\wedge^{s}-m(F)=\lim _{\epsilon \downarrow 0} \wedge^{s}-m_{\epsilon}(F),
$$

and the Hausdorff dimension $H-\operatorname{dim}(F)$ is the supremum $s$ such that $\wedge^{s}-m(F)>0$.

Let $A$ be a subset of $\mathbb{R} \times \mathbb{R}^{d}$. In order to determine if $A$ is polar relative to the heat equation

$$
\sum_{i} \frac{\partial^{2} u}{\partial x_{i}^{2}}=\frac{\partial u}{\partial t}
$$

Taylor and Watson [8] introduced the notion of the restricted Hausdorff dimension of $A$. For any $s>0$, the definition of the restricted Hausdorff measure with index $s$, denoted as $\mathcal{R}-\wedge^{s}-m(A)$, is the same as that for Hausdorff measure except that the balls for covering are replaced by sets of the form

$$
P(t, x ; r)=\left[t, t+r^{2}\right] \times\left[x_{1}, x_{1}+r\right] \times\left[x_{2}, x_{r}+r\right] \times \cdots \times\left[x_{d}, x_{d}+r\right]
$$

where $t \in \mathbb{R}, r \geq 0$ and $x=\left(x_{1}, x_{2}, \cdots, x_{d}\right)$. The restricted Hausdorff dimension of $A$, denoted as $\mathcal{R}-H-\operatorname{dim}(A)$, is defined in terms of the restricted Hausdorff measure in the same way as the Hausdorff dimension is defined in terms of the Hausdorff measure.

We quote a result from Taylor and Watson [8].

Lemma 1. Let $A$ be a Borel subset of $\mathbb{R} \times \mathbb{R}^{d}$ and $s>0$, $c$ be two constants. If $\mu$ is a finite positive measure on $\mathbb{R} \times \mathbb{R}^{d}$ satisfying

$$
\limsup _{r \rightarrow 0} \frac{\mu(A \cap P(t, x ; r))}{r^{s}} \leq c<\infty
$$

for all $(t, x) \in A$, then

$$
\mathcal{R}-\wedge^{s}-m(A) \geq \frac{1}{c 2^{s}} \mu(A) .
$$

Proposition 2. Let $E$ and $F$ be two Borel subsets of $\mathbb{R}$ and $\mathbb{R}^{d}$ respectively. If $0<\wedge^{k}-m(E)<\infty$ and $0<\wedge^{l}-m(F)<\infty$ for some $k, l \geq 0$, then $\mathcal{R}-\wedge^{2 k+l}$ $m(E \times F)>0$.

Proof. This is trivial in the case $k=l=0$. We assume that $k+l>0$. Since $0<\wedge^{k}-m(E)<\infty$ and $0<\wedge^{l}-m(F)<\infty$, it follows from Lemma 5.4 of Hayman and Kennedy [5] that there exist two measures $\mu_{1}$ and $\mu_{2}$ on $\mathbb{R}$ and $\mathbb{R}^{d}$ respectively satisfying the following two conditions:

(1) $0<\mu_{1}(E)<\infty$ and $0<\mu_{2}(F)<\infty$, and

(2) There exists a constant $c$ such that for all $t \in E, x \in F$ and $0<r \leq 1$, we have

$$
\mu_{1}(B(t ; r)) \leq c r^{k}
$$

and

$$
\mu_{2}(B(x ; r)) \leq c r^{l},
$$

where $B(x ; r)$ is the ball centered at $x$ and radius $r$. 
Set $\mu=\mu_{1} \times \mu_{2}$. For every $(t, x) \in E \times F$ and $0<r \leq \frac{1}{\sqrt{d}}$, we have, by the condition (2), that

$$
\begin{aligned}
\mu((E \times F) \cap P(t, x ; r)) & \leq \mu_{1}\left(E \cap\left[t, t+r^{2}\right]\right) \mu_{2}(F \cap B(x ; r \sqrt{d})) \\
& \leq c^{2}\left(\frac{r^{2}}{2}\right)^{k}(r \sqrt{d})^{l}=\frac{c^{2} d^{\frac{l}{2}}}{2^{k}} r^{2 k+l} .
\end{aligned}
$$

Hence

$$
\limsup _{r \rightarrow 0} \frac{\mu((E \times F) \cap P(t, x ; r))}{r^{2 k+l}} \leq \frac{c^{2} d^{\frac{l}{2}}}{2^{k}}<\infty
$$

for all $(t, x) \in E \times F$. It follows from Lemma 1 and condition (1) that

$$
\mathcal{R}-\wedge^{2 k+l}-m(E \times F) \geq \frac{1}{c^{2} d^{\frac{l}{2}} 2^{k+l}} \mu(E \times F)=\frac{1}{c^{2} d^{\frac{l}{2}} 2^{k+l}} \mu_{1}(E) \mu_{2}(F)>0 .
$$

Corollary 3. For every Borel sets $E \subset \mathbb{R}$ and $F \subset \mathbb{R}^{d}$, we have

$$
\mathcal{R}-H-\operatorname{dim}(E \times F) \geq 2 H-\operatorname{dim}(E)+H-\operatorname{dim}(F) .
$$

Proof. Let $k=H-\operatorname{dim}(E)$ and $l=H$ - $\operatorname{dim}(F)$. If $k^{\prime}<k$ and $l^{\prime}<l$, then $\wedge^{k^{\prime}}-(E)=\infty$ and $\wedge^{l^{\prime}}-(F)=\infty$. There exist two Borel subsets $E^{\prime} \subset E, F^{\prime} \subset F$ satisfying $0<\wedge^{k^{\prime}}-m\left(E^{\prime}\right)<\infty$ and $0<\wedge^{l^{\prime}}-m\left(F^{\prime}\right)<\infty$. By Proposition 2, we have

$$
\mathcal{R}-\wedge^{2 k^{\prime}+l^{\prime}}-m(E \times F) \geq \mathcal{R}-\wedge^{2 k^{\prime}+l^{\prime}}-m\left(E^{\prime} \times F^{\prime}\right)>0 .
$$

By the definition of the restricted Hausdorff dimension, we get

$$
\mathcal{R}-H-\operatorname{dim}(E \times F) \geq 2 k^{\prime}+l^{\prime} .
$$

Since this holds for every $k^{\prime}<k$ and $l^{\prime}<l$, we obtain that $\mathcal{R}-H$ - $\operatorname{dim}(E \times F) \geq$ $2 H-\operatorname{dim}(E)+H-\operatorname{dim}(F)$.

Note that $P(t, x ; r) \subset B\left((t, x) ; r \sqrt{d+r^{2}}\right)$ for all $(t, x) \in \mathbb{R}^{d}$ and $r \geq 0$. It follows from the definitions that $\mathcal{R}-\wedge^{s}-m(A) \geq \wedge^{s}-m(A)$ for all $s \geq 0$ and all subsets $A \subset \mathbb{R}^{d+1}$. Hence

$$
\mathcal{R}-H-\operatorname{dim}(A) \geq H-\operatorname{dim}(A) \text { for all } A \subset \mathbb{R}^{d+1} .
$$

The following example is a modification of Example 7.8 in Falconer [4], and it shows that the equality in (4) does not hold for general Borel sets $E$ and $F$.

Example 1. Let $0=a_{0}<a_{1}<a_{2}<\cdots$ be an increasing sequence of integers. Put

$$
\begin{aligned}
E=\left\{r \in[0,1] \mid r=0 . r_{1} r_{2} \cdots r_{i} \cdots,\right. \\
\text { where } \left.r_{i}=0 \text { whenever } a_{2 k}+1 \leq i \leq a_{2 k+1} \text { for some integer } k\right\}
\end{aligned}
$$

and

$$
\begin{aligned}
F_{1}=\{r \in[0,1] \mid r & =0 . r_{1} r_{2} \cdots r_{i} \cdots, \\
& \text { where } \left.r_{i}=0 \text { whenever } a_{2 k+1}+1 \leq i \leq a_{2 k+2} \text { for some integer } k\right\} .
\end{aligned}
$$

It was shown in Falconer [2] that if the $a_{i}$ increase sufficiently rapidly, then $H$ $\operatorname{dim}\left(E \times F_{1}\right) \geq 1$ and $H-\operatorname{dim}(E)=H-\operatorname{dim}\left(F_{1}\right)=0$. Set

$$
F=\left\{\left(x_{1}, 0,0, \cdots, 0\right) \in \mathbb{R}^{d}, \mid x_{1} \in F_{1}\right\} .
$$


The formula for Hausdorff dimension of product sets implies that $H$ - $\operatorname{dim}(F)=$ $H-\operatorname{dim}\left(F_{1}\right)=0$, and $H-\operatorname{dim}(E \times F)=H-\operatorname{dim}\left(E \times F_{1}\right) \geq 1$. It follows from (5) that

$$
\mathcal{R}-H-\operatorname{dim}(E \times F) \geq H-\operatorname{dim}(E \times F) \geq 1>0=2 H-\operatorname{dim}(E)+H-\operatorname{dim}(F) .
$$

To get a sufficient condition for the equality in (4) to hold, we recall a definition of box dimension for subset $F$ of $\mathbb{R}^{d}$. For every $\epsilon>0$, let $N_{\epsilon}(F)$ be the number of $\epsilon$-mesh cubes that intersect $F$. Here an $\epsilon$-mesh cube is one of the form

$$
\left[m_{1} \epsilon,\left(m_{1}+1\right) \epsilon\right] \times \cdots \times\left[m_{d} \epsilon,\left(m_{d}+1\right) \epsilon\right]
$$

where $m_{1}, \ldots, m_{d}$ are integers. The lower and upper box dimensions of $F$ are defined as

$$
B-\operatorname{dim}_{-}(F)=\liminf _{\epsilon \rightarrow 0} \frac{\log N_{\epsilon}(F)}{-\log \epsilon}
$$

and

$$
B-\operatorname{dim}_{+}(F)=\limsup _{\epsilon \rightarrow 0} \frac{\log N_{\epsilon}(F)}{-\log \epsilon} .
$$

The box dimension of $F$ is given by

$$
B-\operatorname{dim}(F)=\lim _{\epsilon \rightarrow 0} \frac{\log N_{\epsilon}(F)}{-\log \epsilon}
$$

(if this limit exists). Note that for every $\epsilon>0$, the $N_{\epsilon}(F)$ number of $\epsilon$-mesh cubes that intersect with $F$ forms a covering for $F$. Hence for every $s>0$, we have

$$
\wedge^{s}-m_{\epsilon \sqrt{d}}(F) \leq(\epsilon \sqrt{d})^{s} N_{\epsilon}(F) .
$$

Taking the logarithm and then dividing by $-\log \epsilon$ (we assume that $\epsilon<1$ ) on both sides gives

$$
s+\frac{s \log d}{2 \log \epsilon}+\frac{\log \left(\wedge^{s}-m_{\epsilon \sqrt{d}}(F)\right)}{-\log \epsilon} \leq \frac{\log N_{\epsilon}(F)}{-\log \epsilon} .
$$

As $\epsilon \downarrow 0$, we get

$$
s+\liminf _{\epsilon \rightarrow 0} \frac{\log \left(\wedge^{s}-m_{\epsilon \sqrt{d}}(F)\right)}{-\log \epsilon} \leq B-\operatorname{dim}_{-}(F) .
$$

If $s<H-\operatorname{dim}(F)$, then $\lim _{\epsilon \rightarrow 0} \wedge^{s}-m_{\epsilon}(F)=\infty$ and hence $s \leq B$ - $\operatorname{dim}_{-}(F)$. Since this holds for all $s<H$ - $\operatorname{dim}(F)$, we observe that

$$
B-\operatorname{dim}_{-}(F) \geq H-\operatorname{dim}(F) .
$$

Although there are many examples in which the above inequality is strict, many reasonably regular sets have the same Hausdorff and box dimension (see Falconer [4] for more details).

Example 2. Let $m$ be a positive integer and $0<\lambda<\frac{1}{m}$. Put $C_{0}=[0,1]$. For $k \geq 0$, assume $C_{k}$ consists of $m^{k}$ closed intervals of lengths $\lambda^{k}$. Then each closed interval $I$ in $C_{k}$ is replaced by $m$ equally spaced subintervals of length $\lambda^{k+1}$, the ends of the $I$ coinciding with the ends of the extreme subintervals. The union of all these subintervals forms the set $C_{k+1}$. Put

$$
C(m, \lambda)=\bigcap_{k} C_{k}
$$


Note that $C\left(2, \frac{1}{3}\right)$ is the middle third Cantor set. Clearly, for every $m$ and $\lambda>$ $0, C(m, \lambda)$ is a set of Lebesgue measure zero. Moreover we have

$$
H-\operatorname{dim}(C(m, \lambda))=B-\operatorname{dim}(C(m, \lambda))=\frac{\log m}{-\log \lambda}
$$

(for a proof, see Falconer [4] Example 4.5)).

Proposition 4. For any sets $E \subset \mathbb{R}$ and $F \subset \mathbb{R}^{d}$, we have

$$
\mathcal{R}-H-\operatorname{dim}(E \times F) \leq 2 H-\operatorname{dim}(E)+B-\operatorname{dim}_{-}(F) .
$$

Proof. Let $k=H-\operatorname{dim}(E)$ and $l=B$ - $\operatorname{dim}_{-}(F)$. Choose $k^{\prime}>k$ and $l^{\prime}>l$. By the definition of box dimension, there exists $\epsilon_{0}>0$ such that

$$
N_{\epsilon}(F) \leq \epsilon^{-l^{\prime}} \text { for all } \epsilon<\epsilon_{0} .
$$

Let $E_{j}$ be any $\epsilon$ - cover of $E$ by intervals with $\sum_{j}\left|E_{j}\right|^{k^{\prime}}<1$. For each $j$, let $F_{j n}$ be the $\sqrt{\left|E_{j}\right|}$-mesh cubes that intersect with $F$. Then $E_{j} \times F_{j n}$ are sets of the form (3) and

$$
\bigcup_{j} \bigcup_{n} E_{j} \times F_{j n}
$$

is a covering of $E \times F$ with $\operatorname{diam}\left(E_{j} \times F_{j n}\right) \leq \sqrt{\epsilon(d+\epsilon)}$. Set $\epsilon^{\prime}=\sqrt{\epsilon+d}$. For $\sqrt{\epsilon}<\epsilon_{0}$, we have

$$
\begin{aligned}
\mathcal{R}-\wedge^{2 k^{\prime}+l^{\prime}}-m \sqrt{\epsilon(d+\epsilon)}(E \times F) & \left.\leq \sum_{j} \sum_{n}\left[\sqrt{\left|E_{j}\right|\left(d+\left|E_{j}\right|\right.}\right)\right]^{2 k^{\prime}+l^{\prime}} \\
& \leq\left(\epsilon^{\prime}\right)^{2 k^{\prime}+l^{\prime}} \sum_{j}\left|E_{j}\right|^{k^{\prime}+\frac{l^{\prime}}{2}}\left|E_{j}\right|^{-\frac{l^{\prime}}{2}} \\
& \leq\left(\epsilon^{\prime}\right)^{2 k^{\prime}+l^{\prime}} \sum_{j}\left|E_{j}\right|^{k^{\prime}}<\infty
\end{aligned}
$$

which implies that $\mathcal{R}-H-\operatorname{dim}(E \times F) \geq 2 k^{\prime}+l^{\prime}$. Since this holds for all $k^{\prime}>k$ and $l^{\prime}>l$, we obtain that $\mathcal{R}-H-\operatorname{dim}(E \times F) \geq 2 H-\operatorname{dim}(E)+B$ - $\operatorname{dim}_{-}(F)$.

Combining Corollary 3 and Proposition 4 we get the following main theorem.

Theorem 5. Let $E \subset \mathbb{R}$ and $F \subset \mathbb{R}^{d}$ be two Borel sets. If $H-\operatorname{dim}(F)=$ $B-\operatorname{dim}(F)$, then we have

$$
\mathcal{R}-H-\operatorname{dim}(E \times F)=2 H-\operatorname{dim}(E)+H-\operatorname{dim}(F) .
$$

Remark. Consider the logarithmic Hausdorff dimension instead (for a definition see, e.g., Dynkin [3]) and define the box dimension of $F$ as

$$
\lim _{\epsilon \rightarrow 0} \frac{\log N_{\epsilon}(F)}{\log (-\log \epsilon)} .
$$

Using the same approach as before, we prove that the restricted logarithmic Hausdorff dimension of $E \times F$ is the sum of the logarithmic Hausdorff dimensions of $E$ and of $F$. 


\section{Applications}

Consider an $(L, \alpha)$-superdiffusion $X$ and assume that $d>\frac{2}{\alpha-1}$. (Note that there is no $\mathbb{G}$-polar set in the case $d<\frac{2}{\alpha-1}$.) Set $\gamma_{0}=d-\frac{2}{\alpha-1}$. Sheu [7] showed that the critical restricted Hausdorff dimension for $\mathbb{G}$-polarity is $\gamma_{0}$. (This means that if $\mathcal{R}-H$ - $\operatorname{dim}(A)<\gamma_{0}$, then $A$ is $\mathbb{G}$-polar; whereas, if $\mathcal{R}-H$ - $\operatorname{dim}(A)>\gamma_{0}$, then it is not $\mathbb{G}$-polar). In fact $\gamma_{0}$ is also the critical Hausdorff dimension for $\mathbb{H}$-polarity in $\mathbb{R}^{d}$ (see, e.g., Dynkin [3] and Sheu [7]). Using these facts and Theorem 5, we obtain the following theorem (which give an answer to Dynkin's problem).

Theorem 6. Assume that $F$ is a Borel subset of $\mathbb{R}^{d}$ and satisfies the condition

$$
\gamma=H-\operatorname{dim}(F)=B-\operatorname{dim}(F)<\gamma_{0} .
$$

Let $E$ be a Borel subset of $\mathbb{R}$. Then

(1) If $H-\operatorname{dim}(E)<\frac{1}{2}\left(\gamma_{0}-\gamma\right)$, then $E \times F$ is a $\mathbb{G}$-polar set.

(2) If $H-\operatorname{dim}(E)>\frac{1}{2}\left(\gamma_{0}-\gamma\right)$, then $E \times F$ is not $\mathbb{G}$-polar.

Remark. (1) In [8], Taylor and Watson also considered polarity of sets of the form $T \times\{0\}, 0 \in \mathbb{R}^{d}, T \subset \mathbb{R}$, for the heat equation. They showed that $T \times\{0\}$ is polar if, and only if $C_{\frac{d}{2}}(T)=0$, where $C_{\beta}$ is the Riesz capacity of order $\beta$. (See also Kaufman and $\mathrm{Wu}[6]$.)

(2) Example 1 shows that there exist Borel sets $E \subset \mathbb{R}$ and $F \subset \mathbb{R}^{d}$ satisfying $\mathcal{R}-H-\operatorname{dim}(E \times F) \geq 1$ and $H-\operatorname{dim}(E)=H-\operatorname{dim}(F)=0$. Clearly $E \times F$ is not $\mathbb{G}$-polar if $d-\frac{2}{\alpha-1}<1$. Therefore the condition that $H$ - $\operatorname{dim}(F)=B$ - $\operatorname{dim}(F)$ is crucial.

Example 3. Let $F=\{0\}$ be the origin point of $\mathbb{R}^{d}$ and $E=C(m, \lambda)$, where $m$ is an integer, $0<\lambda<\frac{1}{m}$ and $C(m, \lambda)$ is defined as in Example 2. Clearly $H-\operatorname{dim}(F)=B-\operatorname{dim}(F)=0$. By Theorem 5 and $(6)$, we have

$$
\mathcal{R}-H-\operatorname{dim}(C(m, \lambda) \times\{0\})=2 H-\operatorname{dim}(C(m, \lambda))=\frac{2 \log m}{-\log \lambda} .
$$

Theorem 6 says that if $m<\lambda^{-\frac{\gamma_{0}}{2}}$, then $C(m, \lambda) \times\{0\}$ is $\mathbb{G}$-polar; whereas, if $m>\lambda^{-\frac{\gamma_{0}}{2}}$, then it is not $\mathbb{G}$-polar.

Example 4. Take $E=C\left(m_{1}, \lambda_{1}\right)$ and $F$ the $d$ copies of $C\left(m_{2}, \lambda_{2}\right)$. Here $m_{1}, m_{2}$ are two integers and $0<\lambda_{i}<\frac{1}{m_{i}}, i=1,2$. By induction and (1.5), (7.8)-(7.9) of Falconer [4], we have

$$
H-\operatorname{dim}(F)=B-\operatorname{dim}(F)=\frac{d \log m_{2}}{-\log \lambda_{2}} .
$$

Theorem 5 implies that

$$
\mathcal{R}-H-\operatorname{dim}(E \times F)=\frac{2 \log m_{1}}{-\log \lambda_{1}}+\frac{d \log m_{2}}{-\lambda_{2}} .
$$

As in Taylor and Watson [8], we assume that

$$
\lambda_{1}=m_{1}^{-\frac{1}{a_{1}}} \text { and } \lambda_{2}=m_{2}^{-\frac{1}{a_{2}}}
$$

for some $0<a_{1}, a_{2}<1$. Then (9) becomes

$$
\mathcal{R}-H-\operatorname{dim}(E \times F)=2 a_{1}+d a_{2} .
$$


(A similar result was also obtained by Taylor and Watson for the case $m_{1}=m_{2}=$ 2.) Under the assumption that $0<a_{2}<1-\frac{2}{d(\alpha-1)}$, we restate Theorem 6 as follows: If $0<a_{1}<\frac{1}{2}\left[d\left(1-a_{2}\right)-\frac{2}{\alpha-1}\right]$, then $E \times F$ is $\mathbb{G}$-polar, and if $a_{1}>\frac{1}{2}\left[d\left(1-a_{2}\right)-\frac{2}{\alpha-1}\right]$, then it is not $\mathbb{G}$-polar.

Remark. Assume that $d=\frac{2}{\alpha-1}$. Then the critical logarithmic Hausdorff dimension for $\mathbb{H}$-polarity is $\frac{1}{\alpha-1}$, and it is equal to the critical restricted logarithmic Hausdorff dimension of $\mathbb{G}$-polarity (see,e.g., Dynkin [3], Sheu [7]). Using these facts and the remark to Theorem 5, we have results similar to Theorem 6.

\section{ACKNOWLEDGMENTS}

I thank the referee and the editor for their useful comments. This research was partially supported by NSC grant 86-2115-M-009-011, Taiwan.

\section{REFERENCES}

1. Baras, P. and Pierre, M., Problems paraboliques semi-linéares avec donnees measures, Applicable Analysis 18 (1984), 111-149. MR 87k:35116

2. Dynkin, E. B., Superdiffusions and parabolic nonlinear differential equations, Ann. of Probab. 20 (1990), 942-962. MR 93d:60124

3. Dynkin, E. B., Superprocesses and partial differential equations, Ann. of Probab. 21 (1993), 1185-1262. MR 94j:60156

4. Falconer, K., Fractal Geometry : Mathematical Foundations aand Applicationss, John Wiley \& Sons Ltd., 1990. MR 92j:28008

5. Hayman, W. K. and Kennedy, P. B., Subharmonic Functions, vol. 1, Academic Press, 1976. MR 57:665

6. Kaufman, R. and Wu, J. M, Parabolic potential theory, J. Differential Equations 43 (1982), 204-234. MR 83d:31006

7. Sheu, Y. C., A Hausdorff measure classification of G-polar sets for superdiffusions, Probab. Theory Relat. Fields 95 (1993), 521-533. MR 94g:60072

8. Taylor, S. J. and Watson, N. A., A Hausdorff measure classification of polar sets for the heat equation, Math. Proc. Camb. Phil. Soc. 97 (1985), 325-344. MR 86m:35077

Department of Applied Mathematics, National Chiao-Tung University, Hsinchu, TaiWAN

Current address: Mathematical Sciences Research Institute, 1000 Centennial Drive, Berkeley, California 94720-5070

E-mail address: ycsheu@nctu.math.edu.tw 